\title{
Using Phenyl Cations as Probes for Establishing Electrophilicity-Nucleophilicity Relations
}

\author{
Valentina Dichiarante, Maurizio Fagnoni and Angelo Albini \\ Dep. Organic Chemistry, University of Pavia, v. Taramelli 10, 27100 Pavia, Italy
}

angelo.albini@unipv.it

SUPPORTING INFORMATION

8 PAGES

NMR Spectra of Compound 4' p. S2

$\begin{array}{ll}\text { Kinetic Data } & \text { p. S4 }\end{array}$ 
NMR Spectra of compound 4'

${ }^{1} \mathrm{H} \mathrm{NMR}\left(\mathrm{CDCl}_{3}\right)$

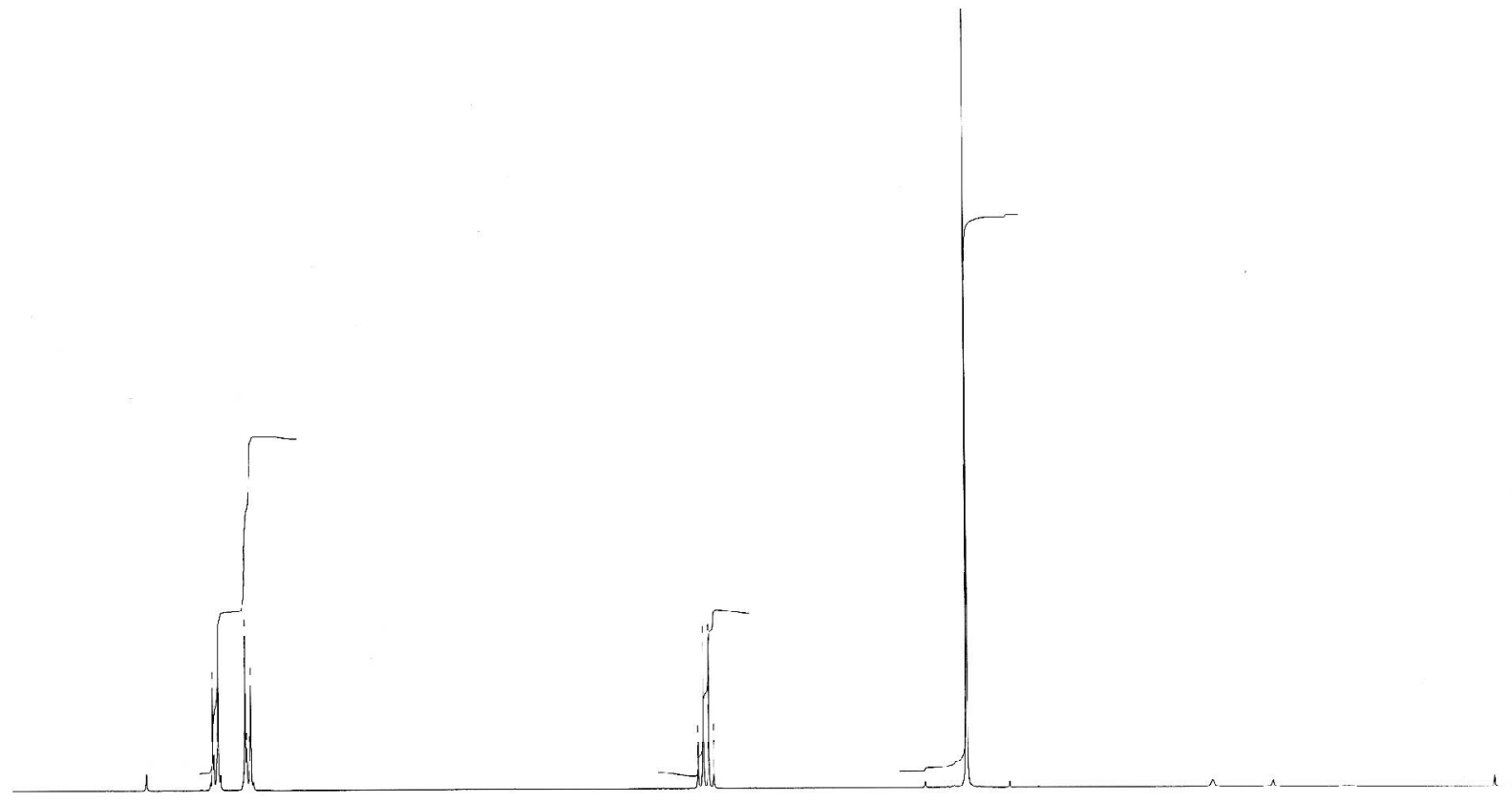

FPm 
${ }^{13} \mathrm{C}$ NMR

言

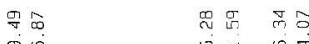

혹 묵



11

i

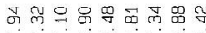

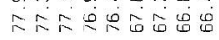

W Y

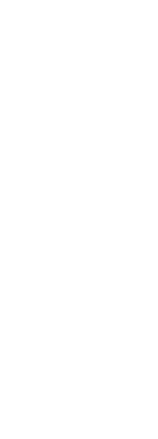

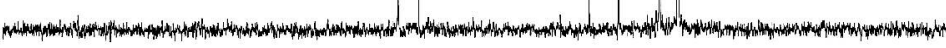

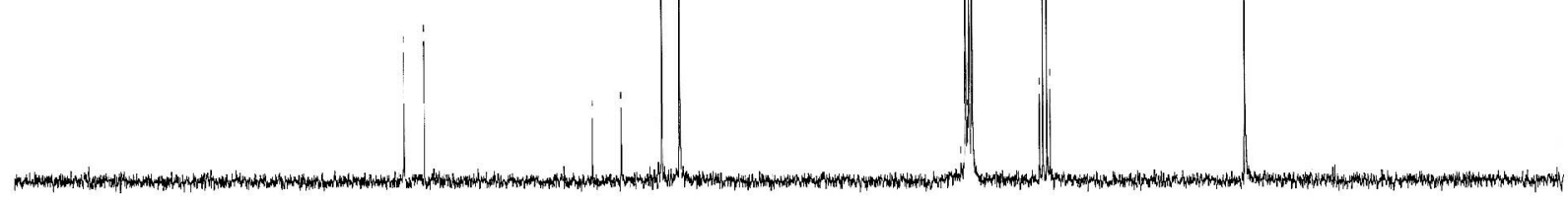

pfm

180

$T_{160}^{T}$

120

80

60

30 


\section{Kinetic Data}

Fluorobenzene, $1 \mathrm{M}$ :

\begin{tabular}{llll}
\hline$[\mathbf{N a I}]$ & {$[\mathbf{A r I}]$} & $\mathbf{1} /[\mathbf{N a I}]$ & $\mathbf{1} /[\mathbf{A r I}]$ \\
\hline 0.05 & 0.00585 & 20 & 170.81 \\
0.02 & 0.00510 & 50 & 195.87 \\
0.015 & 0.00450 & 67 & 222.37 \\
0.01 & 0.00397 & 100 & 251.89 \\
\hline
\end{tabular}

Slope: 1.0374; Intercept: $148.77 ; \mathrm{R}^{2}$ : 0.9886

\section{Chlorobenzene, 1 M:}

\begin{tabular}{llll}
\hline$[\mathbf{N a I}]$ & {$[\mathbf{A r I}]$} & $\mathbf{1} /[\mathbf{N a I}]$ & $\mathbf{1} /[\mathbf{A r I}]$ \\
\hline 0.05 & 0.00538 & 20 & 185.87 \\
0.02 & 0.00452 & 50 & 221.34 \\
0.01 & 0.00330 & 100 & 303.12 \\
0.007 & 0.00290 & 143 & 344.83 \\
\hline
\end{tabular}

Slope: 1.3369; Intercept: $159.18 ; \mathrm{R}^{2}: 0.9901$

Benzene, $1 \mathrm{M}$ :

\begin{tabular}{llll}
\hline$[\mathbf{N a I}]$ & {$[\mathbf{A r I}]$} & $\mathbf{1} /[\mathbf{N a I}]$ & $\mathbf{1} /[\mathbf{A r I}]$ \\
\hline 0.05 & 0.00588 & 20 & 169.92 \\
0.02 & 0.00529 & 50 & 188.95 \\
0.01 & 0.00352 & 100 & 283.61 \\
0.007 & 0.00280 & 143 & 357.47 \\
\hline
\end{tabular}

Slope: 1.5896; Intercept: $125.6 ; \mathrm{R}^{2}$ : 0.9808

1,3-Dimethoxybenzene, $1 \mathrm{M}$ :

\begin{tabular}{llll}
\hline$[\mathbf{N a I}]$ & {$[\mathbf{A r I}]$} & $\mathbf{1} /[\mathbf{N a I}]$ & $\mathbf{1} /[\mathbf{A r I}]$ \\
\hline 0.02 & 0.00358 & 50 & 279.75 \\
0.05 & 0.00506 & 20 & 197.71 \\
0.07 & 0.00520 & 14.3 & 192.26 \\
0.1 & 0.00577 & 10 & 173.29 \\
\hline
\end{tabular}

Slope: 2.4099; Intercept: $156.95 ; \mathrm{R}^{2}$ : 0.9880

Mesitylene, $1 \mathrm{M}$ :

\begin{tabular}{llll}
\hline$[\mathbf{N a I}]$ & {$[\mathbf{A r I}]$} & $\mathbf{1} /[\mathbf{N a I}]$ & $\mathbf{1} /[\mathbf{A r I}]$ \\
\hline 0.05 & 0.00492 & 20 & 203.33 \\
0.02 & 0.00330 & 50 & 303.43 \\
0.01 & 0.00196 & 100 & 510.70 \\
0.007 & 0.00170 & 143 & 589.16 \\
0.005 & 0.00125 & 200 & 800.90 \\
\hline
\end{tabular}

Slope: 3.2694; Intercept: 146.06; R²: 0.9905 
Aniline, 1 M:

\begin{tabular}{llll}
\hline$[\mathbf{N a I}]$ & {$[\mathbf{A r I}]$} & $\mathbf{1} /[\mathbf{N a I}]$ & $\mathbf{1 / [ A r I ]}$ \\
\hline 0.02 & 0.00128 & 50 & 784.12 \\
0.05 & 0.00299 & 20 & 333.92 \\
0.07 & 0.00317 & 14.3 & 315.76 \\
0.1 & 0.00390 & 10 & 256.56 \\
0.2 & 0.00457 & 5 & 219.03 \\
\hline
\end{tabular}

Slope: 12.836; Intercept: $126.95 ; \mathrm{R}^{2}$ : 0.9833

Thiophene, 1 M:

\begin{tabular}{llll}
\hline$[\mathbf{N a I}]$ & {$[\mathbf{A r I}]$} & $\mathbf{1} /[\mathbf{N a I}]$ & $\mathbf{1} /[\mathbf{A r I}]$ \\
\hline 0.05 & 0.00529 & 20 & 189.07 \\
0.03 & 0.00464 & 33 & 215.26 \\
0.02 & 0.00413 & 50 & 242.18 \\
0.01 & 0.00302 & 100 & 331.14 \\
\hline
\end{tabular}

Slope: 1.7609; Intercept: 155.05; $\mathrm{R}^{2}$ : 0.9994

Furan, 1 M:

\begin{tabular}{llll}
\hline$[\mathbf{N a I}]$ & {$[\mathbf{A r I}]$} & $\mathbf{1} /[\mathbf{N a I}]$ & $\mathbf{1} /[\mathbf{A r I}]$ \\
\hline 0.1 & 0.00553 & 10 & 180.93 \\
0.07 & 0.00490 & 14.3 & 204.10 \\
0.05 & 0.00458 & 20 & 218.09 \\
0.03 & 0.00396 & 33 & 252.51 \\
\hline
\end{tabular}

Slope: 2.9617; Intercept: $156.67 ; \mathrm{R}^{2}$ : 0.9767

Pyrrole, 1 M:

\begin{tabular}{llll}
\hline$[\mathbf{N a I}]$ & {$[\mathbf{A r I}]$} & $\mathbf{1} /[\mathbf{N a I}]$ & $\mathbf{1} /[\mathbf{A r I}]$ \\
\hline 0.2 & 0.00317 & 5 & 315.61 \\
0.1 & 0.00207 & 10 & 482.54 \\
0.06 & 0.00133 & 17 & 754.32 \\
0.05 & 0.00115 & 20 & 868.29 \\
\hline
\end{tabular}

Slope: 37.122; Intercept: 122.61; $\mathrm{R}^{2}$ : 0.9990

\section{1-Hexyne, 1 M:}

\begin{tabular}{llll}
\hline$[\mathbf{N a I}]$ & {$[\mathbf{A r I}]$} & $\mathbf{1} /[\mathbf{N a I}]$ & $\mathbf{1} /[\mathbf{A r I}]$ \\
\hline 0.02 & 0.00410 & 50 & 243.56 \\
0.05 & 0.00532 & 20 & 188.02 \\
0.1 & 0.00598 & 10 & 167.13 \\
0.2 & 0.00615 & 5 & 162.58 \\
\hline
\end{tabular}

Slope: 1.8422; Intercept: 151.18; $\mathrm{R}^{2}$ : 0.9974

1-Hexene, $1 \mathrm{M}$ :

\begin{tabular}{llll}
\hline$[\mathbf{N a I}]$ & {$[\mathbf{A r I}]$} & $\mathbf{1 / [ N a I}]$ & $\mathbf{1 / [ A r I ]}$ \\
\hline 0.05 & 0.00449 & 20 & 222.43 \\
0.02 & 0.00350 & 50 & 285.86
\end{tabular}



0.01
0.00202
100
493.97

0.005

0.00130

200

770.87

Slope: 3.1217 ; Intercept: $154.52 ; \mathrm{R}^{2}$ : 0.9921

2,3-Dimethyl-2-butene, $0.5 \mathrm{M}$ :

\begin{tabular}{llll}
\hline$[\mathbf{N a I}]$ & {$[\mathbf{A r I}]$} & $\mathbf{1} /[\mathbf{N a I}]$ & $\mathbf{1 / [ A r I ]}$ \\
\hline 0.015 & 0.00454 & 67 & 220.03 \\
0.02 & 0.00515 & 50 & 194.16 \\
0.05 & 0.00659 & 20 & 151.52 \\
0.1 & 0.00703 & 10 & 142.30 \\
\hline
\end{tabular}

Slope: 1.3821; Intercept: $126.21 ; \mathrm{R}^{2}$ : 0.9966

\section{2-Methyl-2-butene, $1 \mathrm{M}$ :}

\begin{tabular}{llll}
\hline$[\mathbf{N a I}]$ & {$[\mathbf{A r I}]$} & $\mathbf{1 / [ N a I}]$ & $\mathbf{1 / [ \mathbf { A r I } ]}$ \\
\hline 0.01 & 0.00183 & 100 & 546.52 \\
0.02 & 0.00315 & 50 & 317.29 \\
0.05 & 0.00495 & 20 & 201.93 \\
0.1 & 0.00578 & 10 & 172.91 \\
\hline
\end{tabular}

Slope: 4.1928; Intercept: $120.98 ; \mathrm{R}^{2}$ : 0.9962

2-Methyl-1-pentene, $1 \mathrm{M}$ :

\begin{tabular}{llll}
\hline$[\mathbf{N a I}]$ & {$[\mathbf{A r I}]$} & $\mathbf{1} /[\mathbf{N a I}]$ & $\mathbf{1} /[\mathbf{A r I}]$ \\
\hline 0.02 & 0.00274 & 50 & 364.61 \\
0.03 & 0.00344 & 33 & 291.00 \\
0.07 & 0.00509 & 14.3 & 196.46 \\
0.2 & 0.00668 & 5 & 149.70 \\
\hline
\end{tabular}

Slope: 4.8006; Intercept: 127.67; $\mathrm{R}^{2}$ : 0.9986

\section{Allyltrimethylsilane, $0.5 \mathrm{M}$ :}

\begin{tabular}{llll}
\hline$[\mathbf{N a I}]$ & {$[\mathbf{A r I}]$} & $\mathbf{1} /[\mathbf{N a I}]$ & $\mathbf{1} /[\mathbf{A r I}]$ \\
\hline 0.02 & 0.00355 & 50 & 281.80 \\
0.05 & 0.00526 & 20 & 190.27 \\
0.07 & 0.00606 & 14.3 & 164.99 \\
0.1 & 0.00642 & 10 & 155.70 \\
\hline
\end{tabular}

Slope: 3.1818; Intercept: 123.18; $\mathrm{R}^{2}$ : 0.9974

\section{2,3-Dihydrofuran, $1 \mathrm{M}$ :}

\begin{tabular}{llll}
\hline$[\mathbf{N a I}]$ & {$[\mathbf{A r I}]$} & $\mathbf{1} /[\mathbf{N a I}]$ & $\mathbf{1} /[\mathbf{A r I}]$ \\
\hline 0.2 & 0.00512 & 5 & 195.32 \\
0.07 & 0.00336 & 14.3 & 297.71 \\
0.05 & 0.00340 & 20 & 293.68 \\
0.02 & 0.00170 & 50 & 589.77 \\
\hline
\end{tabular}

Slope: 8.6859; Intercept: $150.21 ; \mathrm{R}^{2}$ : 0.9829

2,5-Dimethyl-2,4-hexadiene, 1 M: 


\begin{tabular}{llll}
\hline$[\mathbf{N a I}]$ & {$[\mathbf{A r I}]$} & $\mathbf{1} /[\mathbf{N a I}]$ & $\mathbf{1} /[\mathbf{A r I}]$ \\
\hline 0.02 & 0.00125 & 50 & 800.49 \\
0.05 & 0.00286 & 20 & 350.30 \\
0.07 & 0.00342 & 14.3 & 292.25 \\
0.2 & 0.00437 & 5 & 229.00 \\
\hline
\end{tabular}

Slope: 13.229 ; Intercept: $122.67 ; \mathrm{R}^{2}: 0.9821$

Triethylsilane, $0.5 \mathrm{M}$ :

\begin{tabular}{llll}
\hline$[\mathbf{N a I}]$ & {$[\mathbf{A r I}]$} & $\mathbf{1} /[\mathbf{N a I}]$ & $\mathbf{1} /[\mathbf{A r I}]$ \\
\hline 0.02 & 0.00416 & 50 & 240.25 \\
0.05 & 0.00593 & 20 & 168.66 \\
0.07 & 0.00633 & 14.3 & 158.06 \\
0.1 & 0.00679 & 10 & 147.18 \\
0.2 & 0.00721 & 5 & 138.75 \\
\hline
\end{tabular}

Slope: 2.2849; Intercept: $125.2 ; \mathrm{R}^{2}: 0.9983$

Pyridine, 1 M:

\begin{tabular}{llll}
\hline$[\mathbf{N a I}]$ & {$[\mathbf{A r I}]$} & $\mathbf{1} /[\mathbf{N a I}]$ & $\mathbf{1}[\mathbf{A} \mathbf{A r I}]$ \\
\hline 0.02 & 0.00483 & 50 & 207.09 \\
0.03 & 0.00527 & 33 & 189.73 \\
0.07 & 0.00586 & 14.3 & 170.70 \\
0.1 & 0.00602 & 10 & 166.24 \\
\hline
\end{tabular}

Slope: 1.0204; Intercept: $156.07 ; \mathrm{R}^{2}: 1$

n-Propylamine, 1 M:

\begin{tabular}{llll}
\hline$[\mathbf{N a I}]$ & {$[\mathbf{A r I}]$} & $\mathbf{1} /[\mathbf{N a I}]$ & $\mathbf{1} /[\mathbf{A r I}]$ \\
\hline 0.03 & 0.00518 & 33 & 192.99 \\
0.05 & 0.00570 & 20 & 175.44 \\
0.1 & 0.00622 & 10 & 160.64 \\
0.2 & 0.00644 & 5 & 155.14 \\
\hline
\end{tabular}

Slope: 1.3712; Intercept: $147.74 ; \mathrm{R}^{2}: 0.9988$

Diethylamine, $1 \mathrm{M}$ :

\begin{tabular}{llll}
\hline$[\mathbf{N a I}]$ & {$[\mathbf{A r I}]$} & $\mathbf{1} /[\mathbf{N a I}]$ & $\mathbf{1} /[\mathbf{A r I}]$ \\
\hline 0.02 & 0.00203 & 50 & 493.42 \\
0.05 & 0.00407 & 20 & 245.70 \\
0.1 & 0.00502 & 10 & 199.24 \\
0.2 & 0.00561 & 5 & 178.30 \\
\hline
\end{tabular}

Slope: 7.1712; Intercept: $126.78 ; R^{2}: 0.9857$

Pyrrolidine, 1 M:

\begin{tabular}{llll}
\hline$[\mathbf{N a I}]$ & {$[\mathbf{A r I}]$} & $\mathbf{1} /[\mathbf{N a I}]$ & $\mathbf{1} /[\mathbf{A r I}]$ \\
\hline 0.02 & 0.0009 & 50 & 1054.56 \\
0.05 & 0.0022 & 20 & 450.18 \\
0.07 & 0.0025 & 14.3 & 404.18
\end{tabular}


Slope: 18.146; Intercept: $133.92 ; \mathrm{R}^{2}$ : 0.9921 